\title{
Giant Ovarian Mucinous Borderline Tumors. A Report of Two Cases and Literature Review
}

\author{
Paraskeuas MITRAGKAS ${ }^{\mathrm{a}}$, Eirini SYNEKIDOU ${ }^{\mathrm{b}}$, Konstantinos HATZIMISIOS $^{\mathrm{b}}$ \\ aDepartment of Obstetrics and Gynecology, General Hospital of Kozani, Kozani, Greece

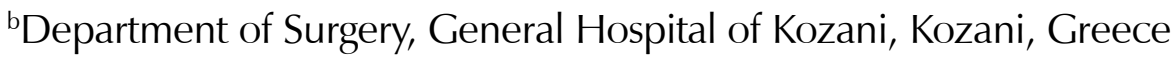

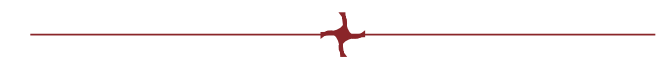

Keywords: adnexa, cancer, gynecology, hysterectomy, neoplasm.

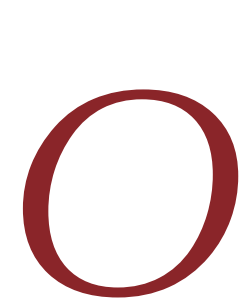

\section{INTRODUCTION}

varian cysts are commonly diagnosed in routine gynecological examinations and they are the main cause of pelvic masses. It is reported that $7 \%$ of women will be diagnosed with an ovarian cyst at some point of their lives (1). In a large screening program, ovarian cysts were detected in $21.2 \%$ of participants. A study screening of 15735 women with trans-vaginal ultrasound revealed one or more ovarian cysts in $14,1 \%$ of participants (2). The advantages of imaging modalities and the establishment of routine examinations have increased the diagnosis of ovarian masses in early stages while they are asymptomatic and small sized. In some rare cases, ovarian masses can remain undiagnosed and grow to huge sizes.

Ovarian cysts that exceed $10 \mathrm{~cm}$ in diameter are called giant ovarian tumors. Herein, we describe two cases of giant ovarian masses treated

\footnotetext{
Address for correspondence:

Paraskeuas Mitragkas

Department of Obstetrics and Gynecology, General Hospital of Kozani, 1 Mamatsiou, 50100 Kozani, Greece

Tel: +00306945055924, email: mitragkas@gmail.com
} 
surgically in our department. In both cases, the final diagnose was giant ovarian mucinous tumor with borderline malignant areas. We do not declare any complication. The following report was written according to Scare guidelines (3).

\section{CASE DESCRIPTION}

\section{CASE 1}

$1 /$ e present the case of a 45-year-old normal cycling woman (gravida 4, para 4) complaining of constipation and early satiety. Her symptoms started gradually the last 12 months. Her past medical and surgical history were unremarkable. On clinical examination, her abdomen was grossly distended and on palpation a painless soft mass was noted. An imaging study with abdominal ultrasound (US) was performed, which revealed a $24 \mathrm{~cm}$ cystic mass. Because the US could not recognize the origin of the mass, a computed tomography (CT) scan of the pelvis and abdomen was performed by us.

The CT scan revealed a $23.5 \times 11 \times 23.6 \mathrm{~cm}$ cyst of the right ovary with septations (Figures 1 and 2). All tumor markers were within normal ranges. The preoperative risk assessment (Risk for Malignancy Index RMI:32) was low for malig-

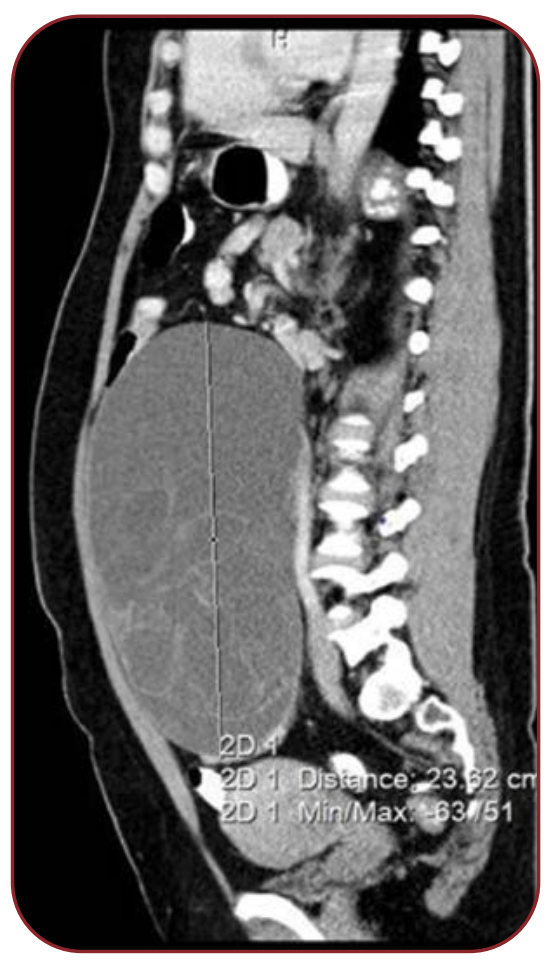

FIGURE 1. CT scan findings of case 1

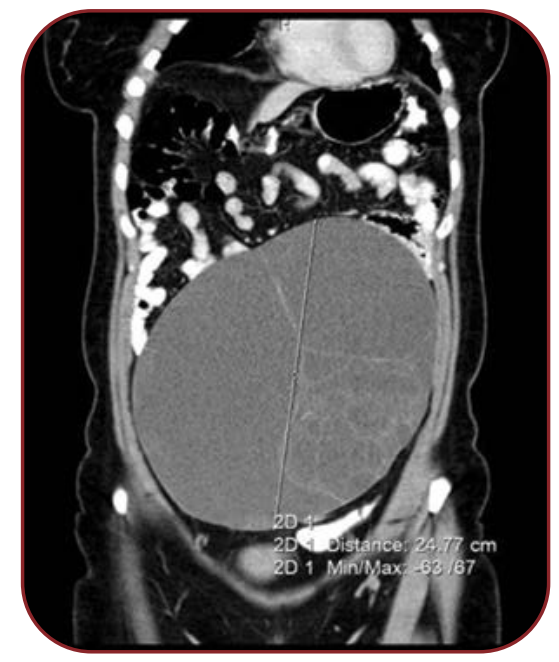

FIGURE 2. CT scan findings of case 1

nancy. A laparotomy with a midline incision was performed. The patient underwent a right adnexectomy without any complication. We managed to remove the mass without any spillage. A thorough surgical exploration was performed without any sign of metastases. The postoperative course was uneventful. The mass was sent for pathology examination. The final diagnosis was a giant mucinous borderline tumor of the right ovary. On the 12-month follow up the patient had normal CT scan and ca125 level.

\section{CASE 2}

The second case was a 53-year-old postmenopausal woman (gravida 2, para 1) who presented with complains of abdominal bloating and epigastrium pain which started the last six months. Her past medical history was remarkable for hypertension. She had undergone a laparoscopic cholecystectomy five years ago. On clinical examination, her abdomen was distended and soft. Giordano, Mc Burney and Murphy signs were negative. During palpation a giant elastic mass was detected. On ultrasound imaging of the abdomen a huge cystic mass of the right ovary was noted. The mass was multiloculated with septations and had intracystic solid areas. All tumor markers were in normal ranges. The preoperative risk assessment (Risk for Malignancy Index RMI: 297) was high for malignancy. We performed a total abdominal hysterectomy with bilateral adnexectomy and omentectomy. The removal of the cyst was uneventful (Figure 3). No 


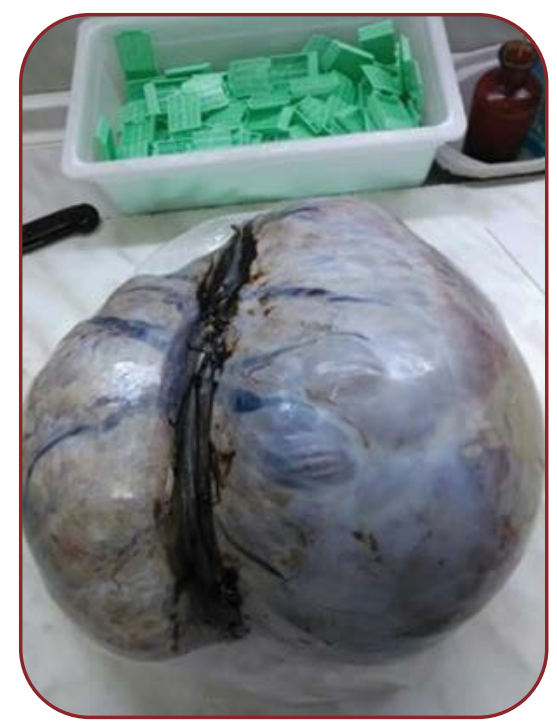

FIGURE 3. Giant ovarian tumor of case 2

signs of extraovarian metastases were revealed from the surgical exploration. The postoperative course was without any complication. The mass was sent for pathology examination. The final diagnosis was a giant mucinous borderline tumor of the right ovary. No signs of recurrence or metastasis were found on the 12-month follow up. $\square$

\section{DISCUSSION}

M ucinous ovarian tumors account for $15 \%$ of all ovarian neoplasms and range from benign cystadenoma to malignant cystadenocarcinoma. They are unilateral with smooth surface and contain viscous fluid (4).

Ovarian cystic masses with $>10 \%$ borderline architecture are categorized as borderline ovarian tumors (BOTs), which are ranked in six subtypes; among those, serous BOTs (SBOTs) $(50 \%)$ and mucinous BOTs (MBOTS) (45\%) are the most common ones, and endometrioid, clear cell and Brenner borderline tumors the less common subtypes.

Mucinous BOTs, also described as atypical proliferative mucinous tumors, are categorized into intestinal type (85\%) and seromucinous type (15\%), which can progress to invasive mucinous carcinoma if untreated (5).

In the literature there are scarce reports of giant MBOTs with a variety of clinical manifestations. The most frequent symptoms are arising from the compression of the neighboring organs such as the bowel, urinary track and veins. Abdominal bloating, constipation, bowel ischemia (6) hydronephrosis (7), cardiac failure and deep venous thrombosis due to reduced venous return (8) have been reported as compressive symptoms. Furthermore, patients with giant MBOTs can present with acute abdomen following torsion or rupture of the cyst (9). But in some cases, the diagnosis is incidental (10).

For the diagnosis an imaging study of the pelvis and abdomen is required. The trans abdomen ultrasound is preferred as a first-line imaging modality (11).

However, more advanced modalities such as CT scans and magnetic resonance imaging (MRI) provide more reliable information about the mass and anatomical structures in close proximity (12).

Tumor markers have been used for calculating the risk of malignancy of the detected ovarian masses, with Ca 125 being the most useful one. The index of malignancy risk, incorporating age, sonographic findings and ca125 level, has a high sensitivity $(74.4 \%)$ and specificity $(90 \%)$ in detecting ovarian cancer in women with adnexal masses (13).

Confirmed prognostic factors comprise a high International Federation of Gynecology and Obstetrics (FIGO) stage, residual tumor, and age. As opposed to ovarian cancer, borderline ovarian tumors are discovered in early stage (FIGO I), limited to the ovary(ies) and have an excellent prognosis. The overall five-year survival is $95-97 \%$ for (FIGO I) tumors and $65-87 \%$ for (FIGO II-III) tumors (14).

Giant ovarian tumors must be excised in order to relive the compressive symptoms, to establish the final diagnosis and to determine the surgical stage. The preferred approach is through a midline incision to prevent spillage in the abdomen. Cystectomy, salpingo- oophorectomy and total hysterectomy with bilateral salpingoophorectomy can be used to treat giant BOTs. Moreover, laparoscopy is proven to be a safe and efficient treatment with reduced postoperative pain and shorter hospital stay than laparotomy (15). Recently, the results from a multicenter study suggested that conservative treatment (fertility sparing) did not increase the recurrence rate and detailed surgical staging (lymphadenectomy, appendectomy, and omentectomy) did not increase the survival rate (16). 


\section{CONCLUSION}

This report presents two women with surgically treated giant BOTs and intents to deep- en the knowledge and raise awareness about this rare condition.

Conflicts of interest: none declared.

Financial support: none declared.

\section{$\boldsymbol{R}_{\text {EFERENCES }}$}

1. Farghaly SA. Current diagnosis and management of ovarian cysts. Clin Exp Obstet Gynecol 2014;41:609-612.

2. Greenlee RT, Kessel B, Williams CR, et al. Prevalence, incidence, and natural history of simple ovarian cysts among women $>55$ years old in a large cancer screening trial.

Am J Obstet Gynecol 2010;202:373.e1-e9.

3. Agha RA, Borrelli MR, Farwana R, et al. The SCARE 2018 statement: Updating consensus Surgical CAse REport (SCARE) guidelines. Int J Surg 2018;60:132-136.

4. Mills AM, Shanes ED. Mucinous Ovarian Tumors. Surg Pathol Clin 2019;12:565-585.

5. Meinhold-Heerlein I, Fotopoulou C, Harter P, et al. The new WHO classification of ovarian, fallopian tube, and primary peritoneal cancer and its clinical implications. Arch Gynecol Obstet 2016;293:695-700.

6. Albers CE, Ranjit E, Sapra A, et al.
Clinician Beware, Giant Ovarian Cysts are Elusive and Rare. Cureus 2020;12:e6753.

7. Kim HY, Cho MK, Bae EH, et al. Hydronephrosis caused by a giant ovarian cyst. Int Braz J Urol 2016;42:848-849.

8. Amadasi A, Andreola S, Bianchi M, et al. Pulmonary thromboembolism secondary to pelvic thrombosis related to giant ovarian tumor. Autops Case Rep 2019;9:e2018061.

9. Ng IAT, Wong JSM, Wong J, et al. Rare case of torsion of giant ovarian mass post-colonoscopy. J Surg Case Rep 2021;2021:rjab070.

10. Pilone V, Tramontano S, Picarelli $P$, et al. Giant mucinous ovarian borderline tumor. A good lesson from an asymptomatic case. Int J Surg Case Rep 2018;50:25-27.

11. Yeika EV, Efie DT, Tolefac PN, Fomengia JN. Giant ovarian cyst masquerading as a massive ascites: a case report.

BMC Res Notes 2017;10:749.
12. Mulita F, Tavlas $P$, Maroulis I. A giant ovarian mass in a 68-year-old female with persistent abdominal pain and elevated serum CA-125 level. Prz Menopauzalny 2020;19:108-110.

13. Gentry-Maharaj A, Burnell M, Dilley J, et al. Serum HE4 and diagnosis of ovarian cancer in postmenopausal women with adnexal masses. Am J Obstet Gynecol 2020;222:56.e1-56.e17.

14. Fischerova D, Zikan $M$, Dundr $P$, Cibula D. Diagnosis, treatment, and follow-up of borderline ovarian tumors. Oncologist 2012;17:1515-1533.

15. Wang X, Li Y. Comparison of perioperative outcomes of single-port laparoscopy, three-port laparoscopy and conventional laparotomy in removing giant ovarian cysts larger than $15 \mathrm{~cm}$. BMC Surg 2021;21:205.

16. Gungorduk K, Asicioglu O, Braicu EI, et al. The Impact of Surgical Staging on the Prognosis of Mucinous Borderline Tumors of the Ovaries: A Multicenter Study. Anticancer Res 2017;37:5609-5616. 